\title{
Screening and identification of a CD44v6 specific peptide using improved phage display for gastric cancer targeting
}

\author{
Dan Zhang ${ }^{1}$, Jin Huang ${ }^{2,3}$, Weiming $\mathrm{Li}^{4}$, Zhiyong Zhang ${ }^{1}$, Meng Zhu' ${ }^{1}$, Yun Feng ${ }^{1}$, Yan Zhao ${ }^{1}$ Yarui Li $^{1}$, \\ Shaoying $\mathrm{Lu}^{4}$, Shuixiang $\mathrm{He}^{1}$
}

${ }^{1}$ Department of Gastroenterology, the First Affiliated Hospital of Xi'an Jiaotong University, Xi'an, China; ${ }^{2}$ School of Electronic Information and Artificial Intelligence, Shannxi University of Science \&Technology, Xi'an, China; ${ }^{3}$ School of Materials Science and Engineering, Shannxi Normal University, Xi'an, China; ${ }^{4}$ Department of Vascular Surgery, the First Affiliated Hospital of Xi'an Jiaotong University, Xi'an, China Contributions: (I) Conception and design: S He, D Zhang, S Lu; (II) Administrative support: S He, D Zhang, S Lu, J Huang; (III) Provision of study materials or patients: D Zhang, J Huang, W Li, Z Zhang, M Zhu, Y Li; (IV) Collection and assembly of data: D Zhang, J Huang, W Li, Z Zhang; (V) Data analysis and interpretation: D Zhang, Y Feng, Y Zhao, S He; (VI) Manuscript writing: All authors; (VII) Final approval of manuscript: All authors.

Correspondence to: Shuixiang He, MD, PhD. Department of Gastroenterology, the First Affiliated Hospital of Xi'an Jiaotong University, Xi'an, China. Email: dyyyjxk@xjtu.edu.cn.

Background: Peptide probes can be applied for biomarker targeting to improve the diagnostic accuracy. Cluster of differentiation 44 (CD44) is up-regulated in gastric cancer (GC). Among all the variants of CD44, CD44v6 is reported the most promising biomarker for GC. The purpose of this study was generating and identification a peptide ligand specific to CD44v6.

Methods: A 12-mer phage peptide library was screened on CD44v overexpressed HEK-293 cells with an improved subtractive method. Five candidate sequences emerged. Candidate phages were selected using enzyme-linked immunosorbent assay and competitive inhibition assays. Then the sequence (designated ELT) was chosen for further study. Its binding affinity and specificity were verified on recombinant protein, GC cells, GC tissues and xenograft models based on BALB/c-nu/nu mice using dissociation constant calculation, immunofluorescence, immunohistochemistry and in vivo imaging separately.

Results: The dissociation constant of ELT with recombinant protein was $611.2 \mathrm{nM}$. ELT stained CD44v overexpressed HEK-293 but not the cell expressing wild-type CD44s. On GC cell lines, ELT co-stained with anti-CD44v6 antibody. ELT binding on tumor tissues significantly increased compared with that of paracancer tissues, also showed a linear positive correlation with CD44v6 expression. ELT specifically accumulated in tumor and eliminated in short time in vivo.

Conclusions: ELT can target GC in vitro and in vivo via CD44v6, indicating its potential to serve as a probe for GC targeting diagnosis and therapy.

Keywords: Peptide probe; CD44v6; gastric cancer (GC); phage display; in vivo imaging

Submitted Dec 31, 2019. Accepted for publication Aug 17, 2020.

doi: 10.21037/atm-19-4781

View this article at: http://dx.doi.org/10.21037/atm-19-4781

\section{Introduction}

Gastric cancer (GC) is an important malignance worldwide for decades. In 2018, more than 1 million new cases and estimated 783,000 deaths occurred, making the disease rank fifth in terms of morbidity and third in terms of mortality (1). GC patients always associate with poor outcome and survival, because traditional examination strategies lack of enough sensitivity and specificity. Prior to architectural changes in gastric mucosa, some relevant molecules alter to promote oncogenesis. As a powerful emerging methodology, molecular imaging can detect both structure and function, provide opportunities to diagnosis cancer at 
early age and improve prognosis of GC patients (2). This strategy has made significant development recently from modern imaging techniques to probe chemistry (3).

The key part of a probe is the ligand that can target cancer cells. Peptide probes was applied to plenty of researches due to the advantages such as molecule specificity, rapid binding kinetics, low toxicity, minimal immunogenicity and feasibility of synthesis (4). Peptide phage display is a powerful strategy of identifying novel ligands. In this high-throughput method, randomized peptides are presented on surface of genetically modified filamentous phages using recombinant DNA technology to form a library $(5,6)$. In biopanning, unrelated phage will be washed away and the phage bind to the targets will be eluted and amplified, that results in the enrichment of the phages with high affinity to the target receptor (7). Some remarkable peptides those derived with phage display, demonstrated outstanding properties for targeting lung, esophagus and colon cancer in clinical studies (8).

Peptide phage library can be screened on multifarious targets, including molecules, cells, organs and living animals. To discover peptide ligands with known target, molecules such as recombinant protein, synthesized nucleic acid and polysaccharide are always applied as screening targets. However, during the process of expression, purification, synthesis and coating, advanced structure of these molecules can be destroyed. The screened peptide may not bind to the target on cells or in vivo. Using cells, organs or living animals can maximize the original structure of target molecule in vivo. Nevertheless, these targets own such complicated constituents that the screened peptide ligand cannot possess a specific known receptor.

Cluster of differentiation 44 (CD44) is a transmembrane glycoprotein. CD44 gene is composed by two group exons: the standard exons (exons 1-5, 16-20) and the variable exons (exons 6-15, also known as exons v1-v10) (9). CD44s means the molecule expressed only by the first group of exons. When variable exons insert between exons 5 and 16, CD44v was formed. As a well-known principle receptor of hyaluronic acid, CD44 plays an important role in adherence to the extracellular matrix, matrix degradation, motility, cell proliferation and survival (10).

CD44 is a well-known GC biomarker. However, because of different researchers and approaches, the presence of CD44s in normal, inflamed gastric mucosa is confusing. It is reported that CD44 is absent in normal epithelium and focally positive in atrophic gastritis, peptic ulcer disease and metaplasia (11-13), whereas some other studies claim that normal mucosa express CD44s $(14,15)$. However, there is a general consensus that GC presents up-regulated CD44 variants, especially CD44v (11-14,16,17). Among all the variants contained variable exons, CD44v6 has the most potential to be a biomarker for GC diagnosis and treatment. CD44v6 expression increases gradually in the progression from regular mucosa to carcinoma in stomach. It was absent in normal mucosa, increasingly expressed in intestinal metaplasia, overexpressed in dysplasia and malignant lesions $(18,19)$. CD44v6 expression also correlated with pathological grade, invasion, lymph node and hematogenous metastasis (20). The evidence above proves that CD44v6 can not only be a biomarker of GC at very early age, but also an indicator for development and outcome of the tumor.

The role of CD44 in cancer makes it an ideal target for cancer diagnosis and therapy. In former researches, various ligands such as hyaluronan and its analogues, antibodies, aptamers and peptide were applied and exhibited potential for cancer therapy. A CD44v6 antibody, bivatuzumab, was examined in clinical trials for its outstanding affinity and specificity. Unfortunately, the trail was terminated because of serious side effect (21). A CD44 specific peptide derived from uPA, was also tested in a trial phase II study (22). Peptides have lower immunogenicity and toxicity than antibodies. Peptides have advantages that they are generally with low toxicity and minimal immunogenicity $(23,24)$. However, peptide specific to $\mathrm{CD} 44 \mathrm{v} 6$ that can be used as probe for diagnosis still needs to be discovered.

Our team used to screen and characterize several peptides targeting cancer. Peptide ligands were screened using phage display biopanned on cancer tissues, cancer cells and recombinant protein (25-28). Even though target molecules expressed on cells can maximize their original structure, receptors of screened peptides were still unknown based on the traditional screen protocol. Using recombinant protein as target, we obtained two CD44 specific peptides, one probed pan-CD44, one aimed CD44v3-v10 $(27,28)$. Then we focus on CD44v6, the optimal CD44 variant as a GC biomarker. We also required target protein expressed on cell to improve binding activity in practice. Nude mouse xenograft models were used to detected the binding, distribution and elimination in vivo, because the models can provide human cancer in animal and suitable for in vivo fluorescence imaging.

An improved phage display method was designed and applied for CD44v6 specific peptide developing. The binding affinity and specificity were confirmed in multiple 
ways in vitro and in vivo, suggesting the potential of the peptide as a novel ligand for detection of GC.

We present the following article in accordance with the ARRIVE reporting checklist (available at http://dx.doi. org/10.21037/atm-19-4781).

\section{Methods}

\section{Cell lines}

The GC cell lines SGC-7901, MKN-28 and MKN-45 were bought from the Cell Bank of Type Culture Collection of the Chinese Academy of Sciences (Shanghai, China). HEK293, the human embryonic kidney cell line was purchased from the American Type Culture Collection (Manassas, VA, USA). The cancer cells were cultured in RPMI 1640 medium (Gibco BRL, Gaithersburg, MD, USA) and HEK-293 cell was cultured in Dulbecco's modified Eagle's medium (Gibco BRL). The medium was supplemented with $10 \%$ fetal bovine serum (FBS; Gibco BRL) and 1\% penicillin/streptomycin. All the four kinds of cell line were maintained at $37^{\circ} \mathrm{C}$ in a $5 \% \mathrm{CO}_{2}$ incubator.

\section{Over expression of CD44v on HEK-293 cells}

The recombinant plasmid contained CD44 was constructed by Genechem Co., Ltd. (Shanghai, China). CD44v3-v10 cDNA, which was obtained in a cDNA library, contained the complete coding region of all the standard exons and v3-v10 exons. Then the cDNA was cloned into EcorI and XhoI sites of the GV-146 plasmid (Genechem), which contained GFP reporter and two individual promoters. HEK-293, the embryo derived cell line, expressed wild type CD44s. We transfected the recombinant CD44v3v10 plasmid and the control vector into HEK-293 using Lipofectamine 2000 Transfection Reagent (Invitrogen, Carlsbad, CA, USA).

\section{Selection of CD44v6 phages}

A commercial PhD-12 Phage Display Peptide Library (New England BioLabs, Beverly, MA, USA, Catalog \#E8111L) was applied. Random linear dodecapeptides were fused to coat protein of M13 phage. The library contains approximately 109 different sequences and 1,011 phages in $10 \mu \mathrm{L}$. A modified screening method based on manufacturer's instructions was applied in this research to obtain phages specific to particular region of target protein. HEK-293 cells were seeded in six-well plates and transfected with CD44v3-v10 plasmid and empty plasmid separately. After transfected for $48 \mathrm{hrs}$, the cells were washed with cold Tris-buffered saline (TBS) and blocked in $3 \% \mathrm{BSA}$ in TBS at $37^{\circ} \mathrm{C}$ for $2 \mathrm{~h}$. More than $1 \times 10^{11}$ phages in the library was added into the well with GV-146 transfected HEK-293 cells, after incubation at $37^{\circ} \mathrm{C}$ for $2 \mathrm{~h}$, the suspension contained unbound phages was removed to the well of CD44v3-v10 over expressed HEK-293 cells. Experienced $1 \mathrm{~h}$ period for phage binding at $37^{\circ} \mathrm{C}$, the cells were then wash with $0.5 \%$ TBST (TBS with $0.5 \% \mathrm{v} / \mathrm{v}$ Tween 20 ) for six times. The bound phages were eluted using $0.2 \mathrm{M}$ glycine- $\mathrm{HCl}(\mathrm{pH} 2.2)$ with $1 \%$ BSA and neutralized with $1 \mathrm{M}$ Tris- $\mathrm{HCl}(\mathrm{pH}$ 9.1). Eluants were collected for the next time panning. In the fourth cycle of screening, bound phages on CD44v3-v10 over expressed cells were competitively eluted using a monoclonal antiCD44v6 antibody (Dingguo Changsheng, Beijing, China) at $15 \mathrm{mg} / \mathrm{mL}$. Without amplification, eluted phages were tittered and 20 clones were picked randomly from the titration plate. Recovery rate was calculated using the formula: recovery rate $=$ output phages/input phages.

All the 20 clones were individually amplified and then dissolved in the iodide buffer $(10 \mathrm{mM}$ Tris- $\mathrm{HCl}, 4 \mathrm{M} \mathrm{NaI}$, $1 \mathrm{mM}$ ethylene diamine tetra-acetic acid) for DNA isolation. With the primer 5'-AGTAGCAGAAGCCTGAAGA-3', phage DNA was analyzed by automated DNA sequencer.

\section{Phage ELISA}

HEK-293 cells were seeded in 96-well plates and transfected with CD44v3-v10 plasmid and empty plasmid separately. $48 \mathrm{~h}$ after transfection, the candidate phages and the unrelated phages (URps) were added $\left(1 \times 10^{10} \mathrm{pfu} /\right.$ well) and incubated with cells at $37^{\circ} \mathrm{C}$ for $1 \mathrm{~h}$. After six times wash, bound phages were probed by a goat anti-M13 major coat protein antibody (1:200 v/v; Santa Cruz), which subsequently incubated with HRP-labeled secondary antibody (1:5,000 v/v; ZSGB-BIO). A color-developing reaction was performed with TMB kit (Bioss, Beijing, China). Optical density of $450 \mathrm{~nm}\left(\mathrm{OD}_{450}\right)$ was taken by a microplate reader (Biotek, Winooski, VT, USA). Selectivity rate was determined with the formula: $\left(\mathrm{OD}_{\mathrm{pv}}-\mathrm{OD}_{\mathrm{bv}}\right) /$ $\left(\mathrm{OD}_{\mathrm{pc}}-\mathrm{OD}_{\mathrm{bc}}\right) \cdot \mathrm{OD}_{\mathrm{pv}}$ and $\mathrm{OD}_{\mathrm{pc}}$ represented $\mathrm{OD}_{450}$ of the wells where phages incubated with CD44v3-v10 over expressed cells and control cells. $\mathrm{OD}_{\mathrm{bv}}$ and $\mathrm{OD}_{\mathrm{bc}}$ indicated background $\mathrm{OD}_{450}$ of two kinds of cells without incubation with phages. 


\section{Peptide synthesis}

The candidate peptides (ELTVMGYYPGMS and THMPSDPGMNLL) and a scrambled peptide (EHDNTGMLYSVD) were both synthesized by China Peptides Co., Ltd. (Suzhou, China). Peptides were purified to greater than purity at $95 \%$ and labeled with FITC, rhodamine $\mathrm{B}$ or biotin at the $\mathrm{N}$-terminus.

\section{Competitive inbibition ELISA}

Recombinant CD44v3-v10 protein $(1 \mu \mathrm{g} /$ well) were coated on ELISA plate and incubated with candidate peptide or control peptide at various concentrations. Then the plate was used for candidate phage binding detection using ELISA as described above. Inhibition ratio was calculated as: $100 \%-100 \% \times\left(\mathrm{OD}_{\mathrm{t}}-\mathrm{OD}_{\mathrm{b}}\right) /\left(\mathrm{OD}_{\mathrm{i}}-\mathrm{OD}_{\mathrm{b}}\right)$. $\mathrm{OD}_{\mathrm{t}}$ and $\mathrm{OD}_{\mathrm{i}}$ indicated $\mathrm{OD}_{450}$ in the wells with or without competitor. $\mathrm{OD}_{\mathrm{b}}$ presented background $\mathrm{OD}_{450}$ in the wells without anti-M13 antibody.

\section{Binding affinity}

Immobilized CD44v3-v10 protein/BSA was incubated with different concentrations of biotin labeled peptides. OD values were obtained as in competitive inhibition research and used for dissociation constant $\left(\mathrm{K}_{\mathrm{d}}\right)$ calculation. The fitting curves, Scatchard plots and $\mathrm{K}_{\mathrm{d}}$ were generated with Origin 6.0.

\section{Immunofluorescence}

GC cells were seeded on coverslips and grow overnight. Forty-eight hours before immunofluorescence test, HEK293 cells were transfected with CD44v3-v10 or empty plasmids. Cells were fixed with $4 \%$ (w/v) paraformaldehyde and blocked by $3 \%$ BSA (w/v). For GC cells, after incubation with a monoclone anti-CD44v6 antibody (1:500 $\mathrm{v} / \mathrm{v}$; Abcam, Cambridge, TX, UK) for $1 \mathrm{~h}$, cells were treated using a Cy3-labeled secondary antibody (Bioss, Beijing, China). Then, $5 \mu \mathrm{M}$ FITC-labeled peptides were utilized for cell binding at $37{ }^{\circ} \mathrm{C}$ for $10 \mathrm{~min}$. HEK-293 cells were incubated with $5 \mu \mathrm{M}$ rhodamine B-labeled peptides. After nuclear staining with DAPI, cells were visualized using fluorescence microscope (Leica, Solms, HE, DE).

\section{Tissue array and immunohistochemistry}

The tissue arrays were purchased from Shanghai Outdo
Biotech Co., Ltd. (Shanghai, China), which contained tissues of $31 \mathrm{GC}$ cases. Samples were harvested from both cancer and corresponding pericarcinous tissues. All of the patients included in the study experienced no prior treatment and signed informed consent. Shanghai Outdo Biotech Co., Ltd. provided ethics approval from the Medical Ethics Committee of Taizhou Hospital for sample collection. All the study was carried out in accordance with Helsinki declaration (as revised in 2013).

The tissue array were deparaffinized and rehydrated. For antigen retrieval, sections were submerged in TE buffer ( $\mathrm{pH} 9.0)$ and heated in microwave. Then $3 \%(\mathrm{v} / \mathrm{v})$ hydrogen peroxide in methanol was utilized for endogenous peroxidase activity elimination. Followed by blocked with $5 \% \mathrm{FBS}$, tissues were treated with $10 \mu \mathrm{M}$ biotin-labeled peptides, the same tissues was incubated with the antiCD44v6 antibody (1:200 v/v) used in immunofluorescence and then a biotin-labeled secondary antibody (Bioss, China). After the biotin interacted with streptavidin-HRP (Bioss, China), a color developing reaction was performed with diaminobenzidine (DAB). After counterstained with hematoxylin, sections were sealed with neutral balsam.

Based on a previously reported method (29), the staining strength was definite quantity, utilizing the immunohistochemistry score (HSCORE). HSCORE of a case was the mean value of 5 random views, which was calculated as the formula that HSCORE $=\sum \mathrm{Pi}(\mathrm{i}+1)$. In the equation, i meant staining intensity $(0$, none; 1 , weak; 2, moderate; 3, strong), and Pi presented positive cells percentage, ranging from $0-100 \%$ ).

\section{In vivo imaging}

$\mathrm{BALB} / \mathrm{c}-\mathrm{nu} / \mathrm{nu}$ mice athymic mice were all male, with the age at $4-5$ weeks and weight at $20-25 \mathrm{~g}$. The mice were provided by the Laboratory Animal Center of Xi'an Jiaotong University (Xi'an, China). For each mouse, $5 \times 10^{6}$ SGC-7901 cells were subcutaneous injected in the axilla to generate tumor xenografts. Then the animal models were raised for about 3 weeks until tumor grow to the volume at $1 \mathrm{~cm}^{3}$. Mice were raised in SPF level animal room, with temperature at $20-28{ }^{\circ} \mathrm{C}$ and humidity at $40-70 \%$. Full nutrition feed and water were sterilized and supplied for the mice freely. All the animal experiments and welfarerelated assessments were approved by the Medical Ethics Committee of medical school of Xi'an Jiaotong University (No. 2016-132). All applicable international, national, and/ or institutional guidelines for the care and use of animals 

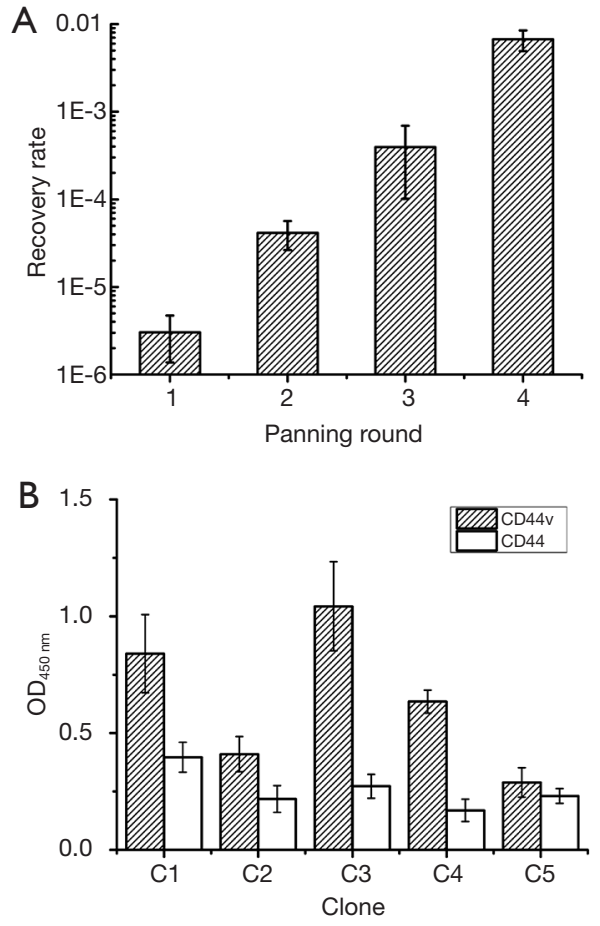

Figure 1 Screening and identification of candidate phage clones. (A) Recovery rate of each panning round; (B) binding of candidate phage clones (presented as OD value at $450 \mathrm{~nm}$ ) to HEK-293 cells transfected with CD44v recombinant plasmid (striped column) and empty plasmid (white column). Data were presented as mean \pm SD. For each data point, measurements were made three times. OD, optical density.

were followed.

Based on former researches (30,31), the animal models were separated into experimental group and control group (ten for each group) with completely random design. At 10 a.m. in laboratory, peptide probe and FITC labeled scrambled peptide were intravenously injected to mice from two groups separately $(1 \mu \mathrm{g} / \mathrm{g}$ body weight). Followed by anesthetized with isoflurane, half of mice were subjected to in vivo fluorescence imaging with IVIS Spectrum Imaging System (Xenogen, Alameda, CA, USA) every half hour until probe was excreted. The other half mice were killed using cervical dislocation when fluorescence signal reached the top in the tumor xenografts. Then tumor tissues and other organs were harvested to analyze biodistribution of peptide probe. Fluorescent intensity for each individual model at each time point was calculated as $\left(\mathrm{p} / \mathrm{sec} / \mathrm{cm}^{2} / \mathrm{sr}\right) /\left(\mu \mathrm{W} / \mathrm{cm}^{2}\right)$ using IVIS Living Imaging 4.2 software.

\section{Statistical analysis}

Statistical analysis was carried out with SPSS 19.0 for Windows (SPSS, Chicago, IL, USA). Date were all expressed as mean $\pm \mathrm{SD}$. The Student's test was applied in quantitative data. For categorical data, the Fisher's exact test was used. Association between two groups of quantitative data was analyzed by the Pearson correlation coefficient. $\mathrm{P}<0.05$ was considered statistically significant.

\section{Results}

\section{Selective panning of CD44v6 expressed on cells}

Considering the significance of CD44v6 on GC diagnosis, a natural large form of the protein CD44v3-10 which containing CD44v6 was chosen. We utilized the recombinant protein with a eukaryotic expression host, human HEK-293 cells for the advanced structure of CD44 on cell surface. Overexpression of CD44v3-v10 was validated using Western blot in our former work (27). For every round of panning, recovery rate (output phages/input phages) increased from $3.05 \times 10^{-6}$ to $6.70 \times 10^{-3}$ (Figure 1A), indicating enrichment of positive clones.

After screening, candidate phages were competitively eluted with anti-CD44v6 antibody and plated. Then 20 clones were randomly picked and sent for DNA sequencing, named C1-C20. All the 20 candidate clones contain five different sequences (Table 1). Among these, sequences of $\mathrm{C} 1$ and $\mathrm{C} 3$ both appeared eight times, while those of $\mathrm{C} 4$ and C5 only emerged one time.

\section{Identification of candidate clones}

Phage ELISA was performed on CD44v3-v10 over expressed HEK-293 cells and wild type HEK-293 cells (control). As shown in Figure $1 B$ and Table 1, OD values of all the five candidate phages in CD44v3-v10 group were significantly different from those in control group $(\mathrm{P}<0.05)$. $\mathrm{C} 3$ and $\mathrm{C} 1$ exhibited the highest binding activity and frequency, as well as a tripeptide motif PGM (Table 1). C3 also showed the second highest selectivity. In this case, C1 and C3 was chosen for peptide synthesis, and named THM and ELT based on the amino acids sequence separately.

\section{Binding of peptides to target protein}

Competition inhibition test was performed to validate that 
Table 1 Amino acid sequences and selectivity of screened peptides

\begin{tabular}{lccc}
\hline Phage clone & AA sequences & Frequency $^{\text {a }}$ & Selectivity $^{2}$ \\
\hline C1 & THMPSDPGMNLL & 2.32 \\
C2 & DVTSNFPHSILR & 2 & 2.22 \\
C3 & ELTVMGYYPGMS & \\
C4 & VAPQSALNMRMA & 8 & 4.65 \\
C5 & SLEVSSWWPLTY & 1 & 5.31 \\
\hline
\end{tabular}

${ }^{\mathrm{a}}$, The number of isolated individual phage clones with the same peptide sequence; ${ }^{\mathrm{b}}$, the sequences with the tripeptide motif PGM.
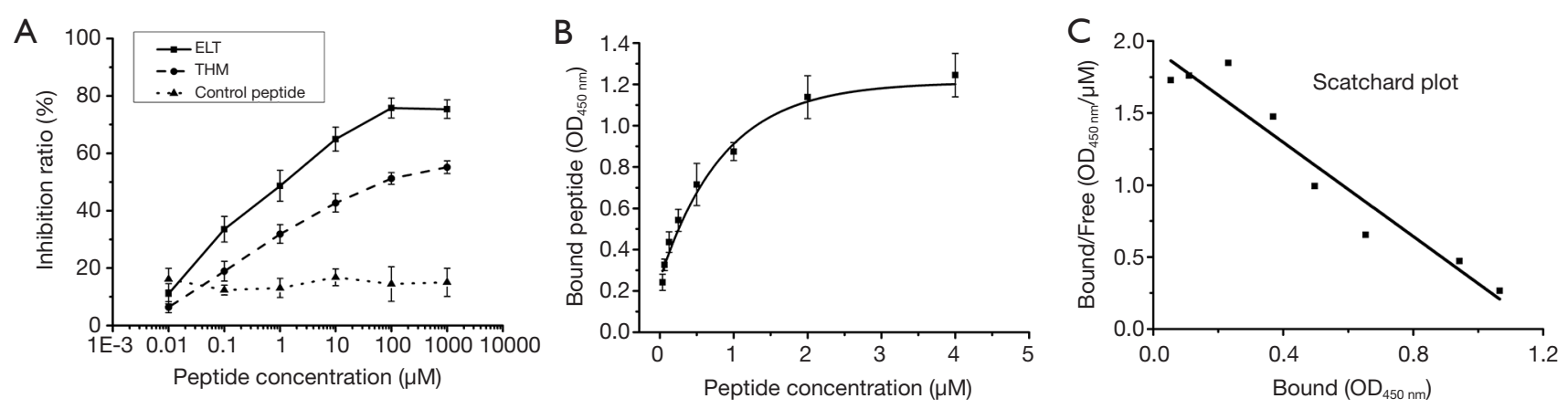

$\mathrm{D}$
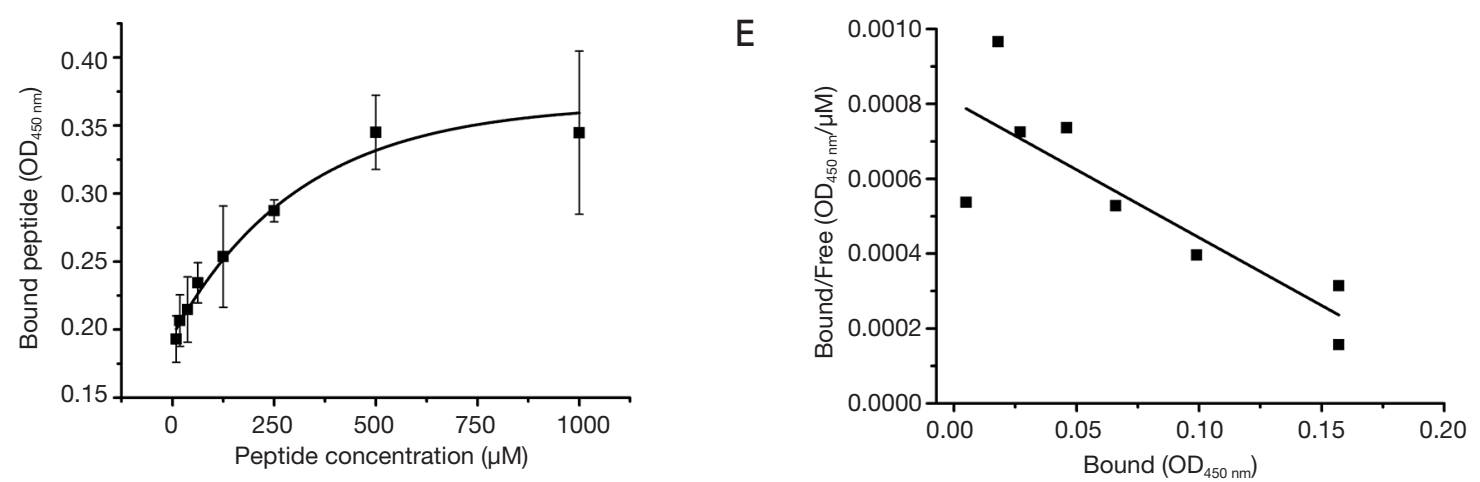

Figure 2 Binding of the candidate peptides to the recombinant CD $44 \mathrm{v}$ protein. (A) Competitive inhibition of candidate phages binding to CD44v protein by corresponding displayed peptide; (B) binding curve of ELT peptide at different concentrations to CD44v protein; (C) Scatchard analysis of ELT binding data with CD44v protein; (D) binding curve of ELT peptide at different concentrations to BSA protein; (E) Scatchard analysis of ELT binding data with BSA protein. A scrambled peptide served as the control. Data were presented as mean \pm SD. For each data point, measurements were made three times.

recombinant phages bound target protein with displayed peptides rather than phage coat protein. For both THM and ELT, inhibition demonstrated as a dose-dependent manner. Inhibition ratio of ELT were higher than that of THM for each concentration of peptide (Figure $2 A$ ). In control group, unrelated phage binding can not be competed by its displayed peptide.

Considering ELT performed higher selectivity and inhibition ratio, the peptide was chosen for protein binding activity determinant. Binding peptide increased with peptide concentration (Figure $2 B$ ). $\mathrm{K}_{\mathrm{d}}$ was $611.2 \mathrm{nM}$, which generated from Scatchard plots (Figure 2C). In BSA group, peptide binding also increased with peptide concentration (Figure $2 D$ ). However, $\mathrm{K}_{\mathrm{d}}$ of ELT peptide binding to BSA was up to $275.5 \mu \mathrm{M}$ (Figure 2E). Thus, ELT could bind to CD44v3-v10 protein with high affinity. 


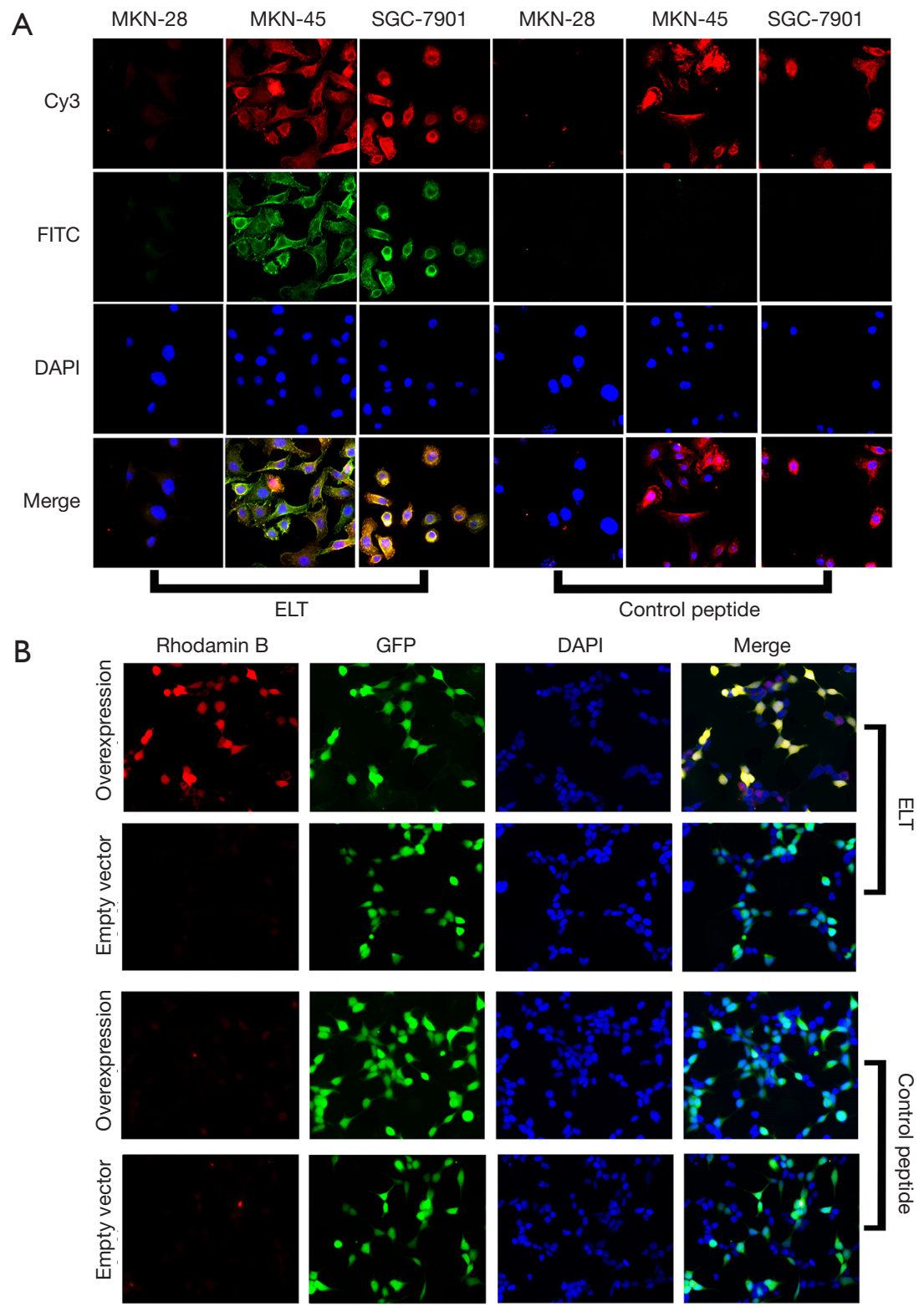

Figure 3 Binding of ELT peptide to GC cells. (A) GC cell lines MKN-28, MKN-45 and SGC-7901 were stained with fluorescence duallabeling technique. Green staining presented FITC-labeled peptide probe binding, while red staining showed CD44v6 expression that was detected by immunofluorescence; (B) binding of rhodamine B labeled peptide probes to CD44v3-v10 recombinant plasmid and empty plasmid. GFP served as a report gene. Cell nuclei were stained with DAPI. Original magnification, $\times 400$. GC, gastric cancer.

\section{Binding of peptide to cells}

Immunofluorescence analysis was performed to evaluate the binding affinity, specificity and binding site of ELT. Three GC cell lines, MKN-45, MKN-28 and SGC-7901 were recruited in the study. Expression of CD44v6 was examined with immunofluorescence. All the three cell lines were all co- stained with CD44v6 antibody and ELT probe (Figure 3A). The results showed that MKN-45 and SGC-7901 expressed CD44v6, but MKN-28 lacked the target protein. The green and red specific fluorescent staining co-located on the cell membrane and cytoplasm of MKN-45 and SGC-7901 in the ELT group. In contrast, significant fluorescence was 
A
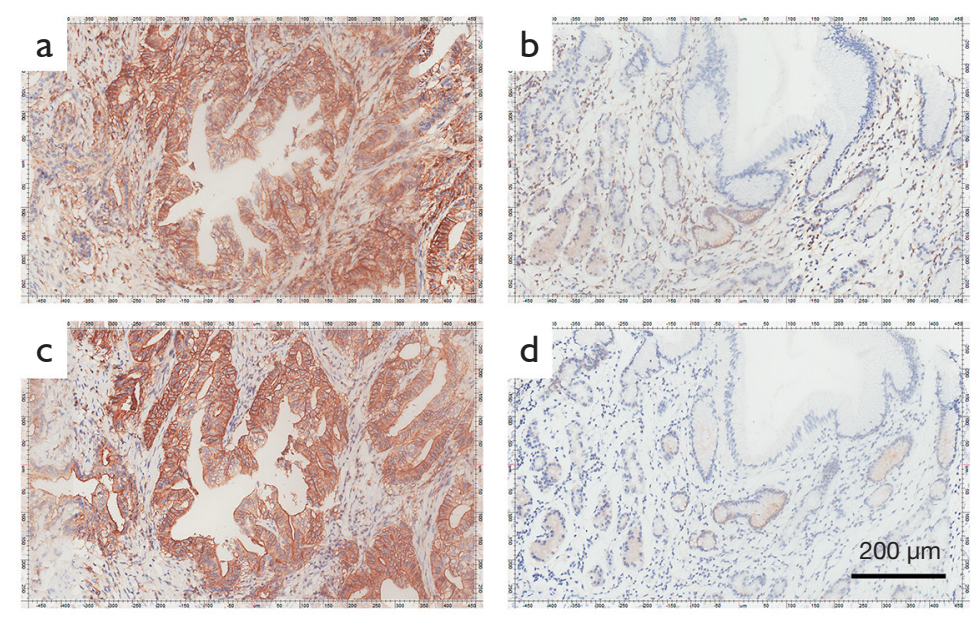

B

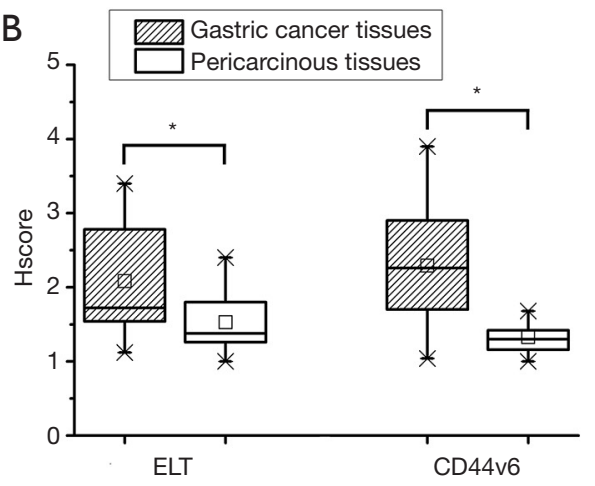

C

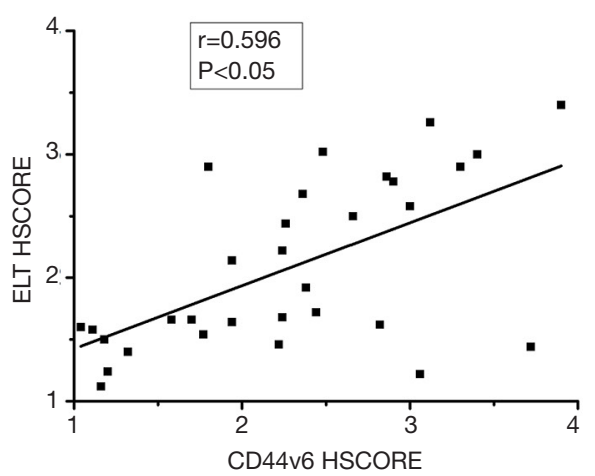

Figure 4 Immunohistochemical staining of ELT and its correlation with CD44v6 expression in gastric tissues (A) Biotin labeled ELT peptide staining and immunohistochemistry detection of CD44v6 on serial sections of GC and adjacent normal tissues from the same patient. (a) ELT stained GC; (b) ELT stained adjacent normal tissue; (c) immunohistochemistry detection of CD44v6 on GC; (d) immunohistochemistry detection of CD44v6 on adjacent normal tissue; (B) statistical analysis of HSCORE of GC tissues (striped box) and pericarcinous tissues (white box) targeted with ELT peptide and anti-CD44v6 antibody. Single asterisk $\left(^{*}\right)$ denotes significant difference; (C) regression line generated using Pearson correlation analysis presents correlation. A linear positive correlation $(r=0.596 \mathrm{P}<0.05)$ was observed between ELT binding and CD44v6 expression. GC, gastric cancer; HSCORE, immunohistochemistry score.

absent on MKN-28 cells. In control peptide group, all the three cell lines showed only background staining.

CD44v3-v10 over expressed HEK-293 cells also been probed with rhodamine B-labeled ELT (Figure 3B). Most red fluorescent staining co-located with GFP. No such correlation was observed in cells transfected with empty plasmids. In control peptide group, only background staining was observed.

\section{Binding of peptide to tissues}

The tissue array contained 31 pairs of gastric carcinoma and their corresponding pericarcinous tissues were utilized for ELT binding test and CD44v6 expression detection. As shown in Figure $4 A$, peptide probe and antibody binding were both located at cytomembrane and cytoplasm. HSCORE of ELT stained GC was $2.09 \pm 0.68$, which decreased to $1.53 \pm 0.38$ in pericarcinous gastric tissue group. HSCORE of CD $44 \mathrm{v} 6$ expression was $2.29 \pm 0.80$ in stomach tumor tissues and $1.33 \pm 0.17$ in adjacent non-tumor tissues. Binding strength of ELT and anti-CD44v6 antibody, quantified with HSCORE, was both significantly different in GC group and adjacent normal tissue group (Figure 4B). Between HSCORE of ELT and CD44v6 stained GC, a linear positive correlation was discovered with Pearson correlation analysis $(\mathrm{r}=0.596 \mathrm{P}<0.05$; Figure $4 C)$. 
A
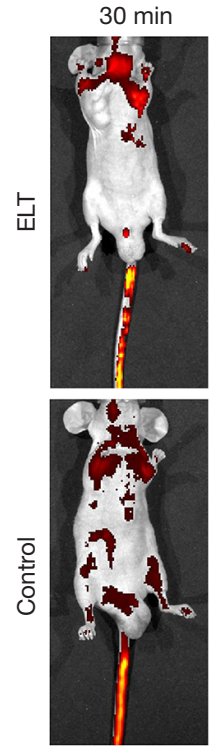

$2 \mathrm{~h}$
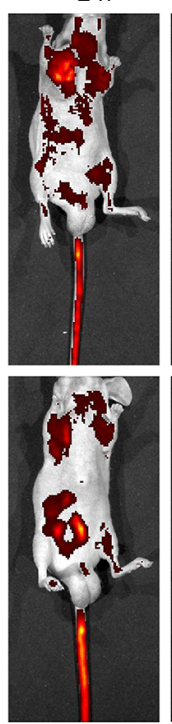

$3 \mathrm{~h}$
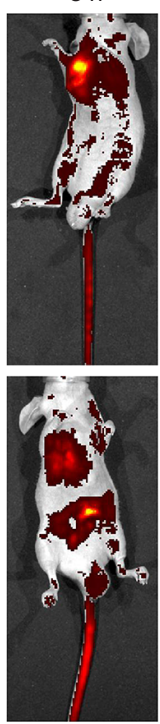

$4 \mathrm{~h}$
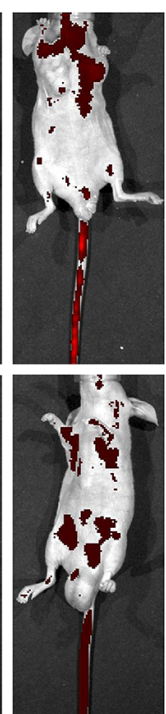

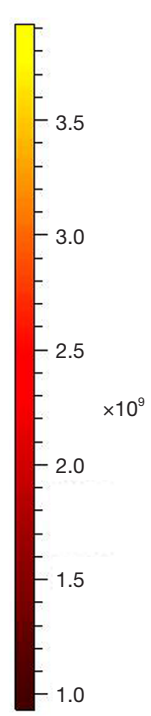

C
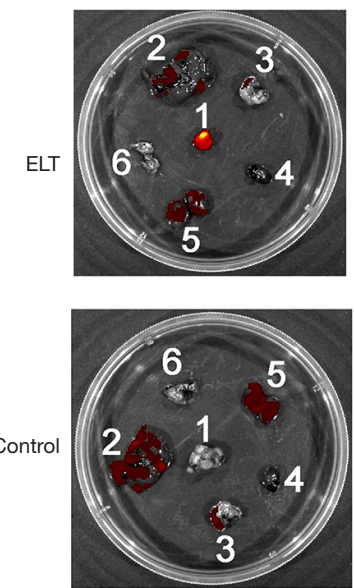

Radiant efficiency $\left(\frac{\mathrm{p} / \mathrm{sec} / \mathrm{cm}^{2} / \mathrm{sr}}{\mu \mathrm{W} / \mathrm{cm}^{2}}\right)$

B
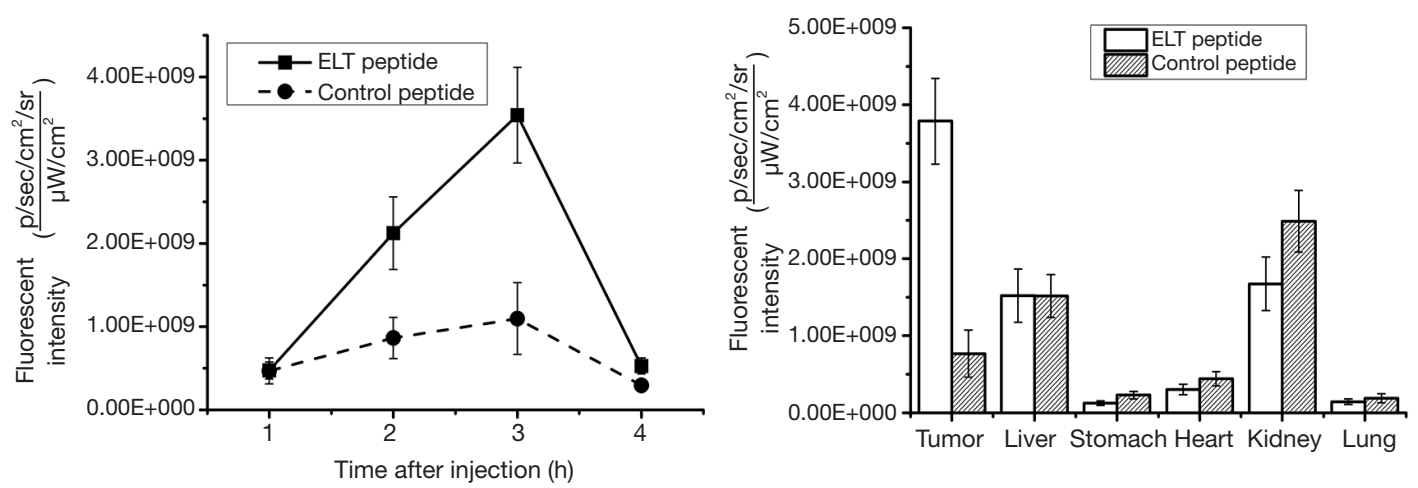

Figure 5 In vivo fluorescence imaging of FITC-ELT probe (A) Serial in vivo fluorescence imaging of FITC labeled ELT and scrambled peptides in nude mice with subcutaneous xenograft tumor at different post-injection time points; (B) fluorescent intensity values of tumor at different time after peptide probe injection. (C) in vivo fluorescence imaging of excised mice organs (1, tumor; 2, liver; 3, stomach; 4, heart; 5, kidney; 6, lung); (D) fluorescent intensity values of excised mice organs. Data were presented as mean \pm SE. For each data point, measurements were made five times.

\section{In vivo imaging}

FITC labeled ELT and control peptide were applied on SGC-7901 subcutaneous GC xenograft models for imaging detection in vivo. Both of peptide probes were injected via tail veins at a dosage of $1 \mu \mathrm{g} / \mathrm{g}$ body weight. Fluorescence images were shot at every half hour after injection. As shown in Figure 5A, ELT probe accumulated in tumor, especially at $3 \mathrm{~h}$ after injection in all five models. Fluorescent intensity reached the top (Figure 5B). No such accumulation of control probe was observed in five models. At $3 \mathrm{~h}$ after injection, tumor tissues and organs (liver, stomach, kidney, lung and heart) were harvested for fluorescence imaging. Strong fluorescence stain were observed on tumor tissue in ELT group (5/5) but not control group (Figure 5C). In both groups, peptides were also distributed in liver and kidney. Fluorescent intensity values in each organ was demonstrated in Figure 5D. The $t$-test on fluorescent intensity of ELT bound tumor and other organs exhibited statistical significance $(\mathrm{P}<0.05)$. Four hours after injection, fluorescence signal was nearly undetectable in both ELT 
and control group, indicating probe had been eliminated from the bodies.

\section{Discussion}

GC is a malignance associated with high mortality due to the difficulty on early diagnosis. Gastroscopy plays a major role on tumor detection. However, the traditional gastroscopy, based on detecting structure abnormality of gastric mucosa, requires experienced examining doctor and accuracy of biopsy. Even if such, high misdiagnosis rate is still existed in practice (32). Molecular imaging techniques can display molecular and functional alteration, providing opportunities to improve the diagnosis rate of early GC. This technique was based on imaging device and contrast agent with specific ligand. PET/SPECT, MR imaging can show tumors and metastases in whole body with systemic administration. For gastrointestinal neoplasms, Endoscopy emerged as a new molecular imaging device with real time performance and high resolution (2). Using a peptide probe topically, Barrett's esophagus can be detected in vivo through gastroendoscopy.

Since scientists have won great success on imaging techniques developing, probes targeting a disease-specific biomarker can contribute to accurate diagnosis by molecular imaging. As reported in plenty of former researches, CD44 is an ideal GC biomarker. A6 peptide was an octapeptide derived from uPA. It was reported to bind the hyaluronic acid binding domain of CD44 and be a ligand for panCD44 (33). A6 peptide could not only bind to CD44 positive ovarian cancer cells, but also inhibit CD44 related malignant behaviors (33). Our team used to screen a CD44 specific peptide and verify the specificity and affinity $(28,31)$. CD44s, the CD44 molecule encoded by only standard exons, can be expressed by normal cells. To the contrary, region of CD44 encoded by variable exons maintains high cancer specificity. To improve diagnosis accuracy, we also screened a peptide targeting v3-v10 exons encoded region with an improved biopanning strategy (27). Considering most reported researches suggested the high sensitivity and specificity of $\mathrm{v} 6$ exon for GC, we aimed to identify peptide targeting this region. Two peptides, derived from CD44v6 amino acid sequence, were reported to block activation of Met, because CD44v6 was assumed as a co-receptor of Met (34). The reported peptides was focused on cancer treatment with CD44 related pathway but not potential as a probe for diagnosis.

As a powerful tool for peptide generating, phage display can be utilized on multifarious targets, including molecules, cells, organs and living animals. In the former work, we screened phage library on recombinant protein to insure the target molecule. However, the advanced structure of protein can not maintain the original form during purification and coating. In this study, we devise a screening strategy to ensure both target protein activity and screening specificity. We chose HEK-293 cell because the cell line expressed CD44s and was suitable for protein over expression. A natural form of CD44 (NM 001001389.1) was used to construct the overexpression vector, because it was the smallest natural homo sapien CD44 variant. HEK-293 cells transfected with empty vector plasmid were applied for negative absorption. The unbound phages were utilized to screen on CD44v overexpressed HEK-293 cells. In this way, phages having affinity with HEK-293 cells and GFP can be eliminated; phages target overexpressed CD44v can be enriched. All the target protein were expressed on cells and maintained advance structure to the maximum extent. To generate phages specific to only CD44v6, we adopt competitive elution strategy using CD44v6 monoclone antibody. In this way, phages targeted the same antigenic determinant on CD44v6 can be eluted. Titer of output phages gradually raised in each round of panning with maintained input phage number. Thus, with the modified panning strategy, positive phages obviously enriched.

Twenty clones, named C1-C20 were randomly selected, followed by phage DNA sequencing. Five individual sequences emerged in the 20 clones, among these, sequences displayed by $\mathrm{C} 1$ and $\mathrm{C} 3$ clone both occurred eight times, the other three clones only appeared no more than two times. Results showed that $\mathrm{C} 1$ and $\mathrm{C} 3$ sequences exhibited the highest potential for target binding. When all the five sequences were applied in ELISA, C3 clone exhibited the highest affinity, while $\mathrm{C} 1$ clone also showed considerable affinity. Thus, both $\mathrm{C} 1$ and $\mathrm{C} 3$ clone were chosen for peptide synthesis. Binding of the two phage clones were competed by their displayed peptide. Inhibition rate increased in peptide dose dependent manner for both clones, indicating phages bound to target via their displayed peptide rather than M13 coat protein. In competitive assay, $\mathrm{C} 3$ clone showed higher inhibition rate than $\mathrm{C} 1$ clone. In sum, C3 sequence exhibited the highest affinity and specificity, and was chosen for further examination.

Binding affinity and specificity of ELT peptide to CD44v6 were verified on molecule, cell, tissue and animal levels. Although ELT was screened on cells, its dissociation constant with recombinant protein was $611.2 \mathrm{nM}$, indicating 
considerable binding affinity. Immunofluorescence colocalization analysis was performed on transfected HEK293 cells and three different GC cell lines. Results showed that ELT only bind to CD44v6 overexpressed HEK-293 cells and CD44v6 positive GC cells, implying the binding specificity to CD44v6. Further, CD44v6 expression and ELT binding, was detected on 31 pairs of GC and their corresponding pericarcinous tissues. In accordance with the former studies $(11,14)$, HSCORE of tumors significantly increased compared with that of paracancer tissues. HSCORE of ELT exhibited the same trend in tissues, and a linear positive correlation with CD44v6 expression, indicating that ELT can detect gastric carcinomas via CD44v6. At last, peptide probe was examined in vivo. Binding conditions in vivo were more complicated than that in vitro. Distribution, metabolism, elimination, halflife of the peptide probe could be affected by multiple factors, including peptide characters, tissue affinity and animal individual difference. At specific time point, ELT specifically accumulated in tumor more than control peptide, distributed in tumor more than other normal organs and eliminated by animal models in short time. As such, ELT is an ideal probe for clinical practice.

CD44 is considered as a valuable biomarker for cancer target diagnosis and treatment. For GC, CD44v6 exhibited the highest potential due to its sensitivity and specificity. CD44v6 antibody was used to be a promising agency for cancer therapy, but the drug failed in clinic trial for its serious side effect. Compared to antibodies, peptides own less amino acid and lower molecular weight. Although some of them showed significant toxic effects, most of have minimal immunogenicity and toxicity in general $(23,24)$. A CD44v6 ligand peptide might target GC specifically and trigger less adverse drug events than antibodies. The followup work will focus on toxicity to confirm our conjecture. Xenograft model for in situ GC will be applied for diagnosis with endoscopy.

\section{Conclusions}

In this study, ELT was screened with a modified protocol and characterized in multiple levels. Binding affinity and specificity of ELT to GC cells, tissues and xenograft in vivo suggested the peptide a potential tool for detection, monitoring and drug delivery for CD44v6 positive tumors. In further studies, we will enlarge the sample size to evaluate peptide detection to GC and precancerous conditions, as well as relationship with disease prognosis. Toxicity and therapeutic action will also be examined, making ELT more applicable in diagnosis and treatment of cancer.

\section{Acknowledgments}

Funding: This work was supported by grants from the National Natural Science Foundation of China (grant numbers 81602611, 81802416, 81472747 and 61904100).

\section{Footnote}

Reporting Checklist: The authors have completed the ARRIVE reporting checklist. Available at http://dx.doi. org/10.21037/atm-19-4781

Data Sharing Statement: Available at http://dx.doi. org/10.21037/atm-19-4781

Peer Review File: Available at http://dx.doi.org/10.21037/ atm-19-4781

Conflicts of Interest: All authors have completed the ICMJE uniform disclosure form (available at http://dx.doi. org/10.21037/atm-19-4781). The authors have no conflicts of interest to declare.

Ethical Statement: The authors are accountable for all aspects of the work in ensuring that questions related to the accuracy or integrity of any part of the work are appropriately investigated and resolved. The study was conducted in accordance with the Declaration of Helsinki (as revised in 2013). All of the patients included in the study experienced no prior treatment and signed informed consent. Shanghai Outdo Biotech Co., Ltd. provided ethics approval from The Medical Ethics Committee of Taizhou Hospital for sample collection. All the animal experiments and welfare-related assessments were approved by the Medical Ethics Committee of Medical School of Xi'an Jiaotong University (No. 2016-132). All applicable international, national, and/or institutional guidelines for the care and use of animals were followed.

Open Access Statement: This is an Open Access article distributed in accordance with the Creative Commons Attribution-NonCommercial-NoDerivs 4.0 International License (CC BY-NC-ND 4.0), which permits the noncommercial replication and distribution of the article with the strict proviso that no changes or edits are made and the 
original work is properly cited (including links to both the formal publication through the relevant DOI and the license). See: https://creativecommons.org/licenses/by-nc-nd/4.0/.

\section{References}

1. Bray F, Ferlay J, Soerjomataram I, et al. Global cancer statistics 2018: GLOBOCAN estimates of incidence and mortality worldwide for 36 cancers in 185 countries. CA Cancer J Clin 2018;68:394-424.

2. Goetz M, Wang TD. Molecular imaging in gastrointestinal endoscopy. Gastroenterology 2010;138:828-33.e1.

3. Joshi BP, Wang TD. Exogenous molecular probes for targeted imaging in cancer: focus on multi-modal imaging. Cancers (Basel) 2010;2:1251-87.

4. Atreya R, Goetz M. Molecular imaging in gastroenterology. Nat Rev Gastroenterol Hepatol 2013;10:704-12.

5. Smith GP. Filamentous fusion phage: novel expression vectors that display cloned antigens on the virion surface. Science 1985;228:1315-7.

6. Noren KA, Noren CJ. Construction of high-complexity combinatorial phage display peptide libraries. Methods 2001;23:169-78.

7. Scott JK, Smith GP. Searching for peptide ligands with an epitope library. Science 1990;249:386-90.

8. Joshi BP, Wang TD. Targeted optical imaging agents in cancer: focus on clinical applications. Contrast Media Mol Imaging 2018;2018:2015237.

9. Goodison S, Urquidi V, Tarin D. CD44 cell adhesion molecules. Mol Pathol 1999;52:189-96.

10. Wang W, Dong LP, Zhang N, et al. Role of cancer stem cell marker CD44 in gastric cancer: a meta-analysis. Int J Clin Exp Med 2014;7:5059-66.

11. Mayer B, Jauch KW, Gunthert U, et al. De-novo expression of CD44 and survival in gastric cancer. Lancet 1993;342:1019-22.

12. Washington K, Gottfried MR, Telen MJ. Expression of the cell adhesion molecule CD44 in gastric adenocarcinomas. Hum Pathol 1994;25:1043-9.

13. Wang T, Ong CW, Shi J, et al. Sequential expression of putative stem cell markers in gastric carcinogenesis. Br J Cancer 2011;105:658-65.

14. Higashikawa K, Yokozaki H, Ue T, et al. Evaluation of CD44 transcription variants in human digestive tract carcinomas and normal tissues. Int J Cancer 1996;66:11-7.

15. Sneath RJ, Mangham DC. The normal structure and function of CD44 and its role in neoplasia. Mol Pathol 1998;51:191-200.
16. Harn HJ, Ho LI, Chang JY, et al. Differential expression of the human metastasis adhesion molecule CD44V in normal and carcinomatous stomach mucosa of Chinese subjects. Cancer 1995;75:1065-71.

17. Kurozumi K, Nishida T, Nakao K, et al. Expression of CD44 variant 6 and lymphatic invasion: importance to lymph node metastasis in gastric cancer. World J Surg 1998;22:853-7; discussion 857-8.

18. da Cunha CB, Oliveira C, Wen X, et al. De novo expression of $\mathrm{CD} 44$ variants in sporadic and hereditary gastric cancer. Lab Invest 2010;90:1604-14.

19. Gulmann C, Grace A, Leader M, et al. CD44v6: a potential marker of malignant transformation in intestinal metaplasia of the stomach? An immunohistochemical study using tissue microarrays. Eur J Gastroenterol Hepatol 2003;15:981-6.

20. Jang BI, Li Y, Graham DY, et al. The role of CD44 in the pathogenesis, diagnosis, and therapy of gastric cancer. Gut Liver 2011;5:397-405.

21. Orian-Rousseau V, Ponta H. Perspectives of CD44 targeting therapies. Arch Toxicol 2015;89:3-14.

22. Gold MA, Brady WE, Lankes HA, et al. A phase II study of a urokinase-derived peptide (A6) in the treatment of persistent or recurrent epithelial ovarian, fallopian tube, or primary peritoneal carcinoma: a Gynecologic Oncology Group study. Gynecol Oncol 2012;125:635-9.

23. Klenske E, Neurath MF, Atreya R, et al. Molecular imaging in gastroenterology: A route for personalized endoscopy. Dig Liver Dis 2018;50:878-85.

24. Muguruma N, Miyamoto H, Okahisa T, et al. Endoscopic molecular imaging: status and future perspective. Clin Endosc 2013;46:603-10.

25. Zhang WJ, Sui YX, Budha A, et al. Affinity peptide developed by phage display selection for targeting gastric cancer. World J Gastroenterol 2012;18:2053-60.

26. Guo Y, Ma C, Li C, et al. Screening and identification of a specific peptide binding to hepatocellular carcinoma cells from a phage display peptide library. J Pept Sci 2014;20:196-202.

27. Zhang $\mathrm{D}$, Jia H, Li W, et al. Screening and Identification of a Phage Display Derived Peptide That Specifically Binds to the CD44 Protein Region Encoded by Variable Exons. J Biomol Screen 2016;21:44-53.

28. Zhang D, Jia H, Wang Y, et al. A CD44 specific peptide developed by phage display for targeting gastric cancer. Biotechnol Lett 2015;37:2311-20.

29. Budwit-Novotny DA, McCarty KS, Cox EB, et al. Immunohistochemical analyses of estrogen receptor in 
endometrial adenocarcinoma using a monoclonal antibody. Cancer Res 1986;46:5419-25.

30. Kim S, Kim D, Jung HH, et al. Bio-inspired design and potential biomedical applications of a novel class of high-affinity peptides. Angew Chem Int Ed Engl 2012;51:1890-4.

31. Li W, Jia H, Wang J, et al. A CD44-specific peptide, RP1 , exhibits capacities of assisting diagnosis and predicting prognosis of gastric cancer. Oncotarget 2017;8:30063-76.

32. Takao M, Kakushima N, Takizawa K, et al. Discrepancies in histologic diagnoses of early gastric cancer between

Cite this article as: Zhang D, Huang J, Li W, Zhang Z, Zhu M, Feng Y, Zhao Y, Li Y, Lu S, He S. Screening and identification of a CD44v6 specific peptide using improved phage display for gastric cancer targeting. Ann Transl Med 2020;8(21):1442. doi: 10.21037/atm-19-4781 biopsy and endoscopic mucosal resection specimens. Gastric Cancer 2012;15:91-6.

33. Piotrowicz RS, Damaj BB, Hachicha M, et al. A6 peptide activates CD44 adhesive activity, induces FAK and MEK phosphorylation, and inhibits the migration and metastasis of CD44-expressing cells. Mol Cancer Ther 2011;10:2072-82.

34. Matzke A, Herrlich P, Ponta H, et al. A five-amino-acid peptide blocks Met- and Ron-dependent cell migration. Cancer Res 2005;65:6105-10. 